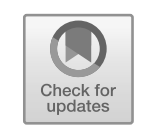

\title{
9 Die Umsetzung: Entscheidungsstrukturen und Anforderungen an Hochschulen
}

Die vorangehenden Kapitel haben darauf fokussiert, was strukturelle Studierbarkeit ist und welche Anforderungen Studierende an die Struktur ihres Studiengangs stellen. Diese Ergebnisse sind jedoch nur dann für die Praxis hilfreich, wenn sie von der Politik und den Entscheidungsträgern in den Hochschulen rezipiert und umgesetzt werden können. In diesem Kapitel steht daher die Umsetzbarkeit der Konzepte im Mittelpunkt. Ob und von wem die Ergebnisse umgesetzt werden können, hängt von Entscheidungsstrukturen in Hochschulen ab. Die Funktionsweise von Organisationen und ihre Steuerung bezogen auf Studium und Lehre wird daher in Kapitel 9.1 analysiert. Im Anschluss erfolgt eine Analyse, die untersucht, welche Anforderungen und Interessen die Hochschulen und ihre Mitglieder (Professorinnen und Professoren, Mitarbeitende) bezogen auf die einzelnen studienstrukturellen Elemente haben.

\subsection{Entscheidungsstrukturen an Hochschulen}

Die Entscheidungsstrukturen und Funktionsweisen von Hochschulen spielen eine wichtige Rolle bei der Frage, wie Veränderungen in Studium und Lehre angestoßen und umgesetzt werden. Wenn eine hohe strukturelle Studierbarkeit erreicht werden soll, sind hierfür in der Regel Anpassungen der Studienstrukturen notwendig. Dieses Kapitel analysiert, wer treibende Kräfte und Bedenkenträger bei diesen Entwicklungen sein können. Dabei sind die Verteilung von Entscheidungskompetenzen in der Hochschule und die Gründe für eine Veränderungsbereitschaft von Lehrenden und Hochschulleitung besonders bedeutsam. Drei Fragenkomplexe sind nachfolgend relevant: erstens die Funktionsweise von Hochschulen, zweitens deren Steuerung und drittens die Beweggründe für Veränderungen in Struktur und Handeln. Diese Aspekte werden von umfangreicher Literatur beleuchtet. Die relevantesten Theorien in diesem Zusammenhang werden im Folgenden und in Tabelle 35 dargestellt und in Bezug auf ihre Relevanz für Entscheidungen zur Studienreform analysiert. 
Tabelle 35 Wichtige Theorien der Hochschulsteuerung.

\begin{tabular}{ll}
\hline Frage & Theorien \\
\hline Wie funktioniert die Organisation & Theorie der lose gekoppelten Systeme \\
Hochschule? & nach \\
& Weick (1976) \\
& Theorie der organisierten Anarchien nach \\
& Cohen et al. (1972) \\
\hline Wie werden Hochschulen & Equalizer-Modell nach Schimank, in: \\
gesteuert? & Beor et al. (2010) \\
\hline Woran orientieren sich die & Soziologische neoinstitutionalistische \\
Organisation und die Mitglieder & Organisationstheorie (DiMaggio und \\
von Hochschulen in Struktur und & Powell 1983; Hasse und Krücken 2005; \\
Handeln? & Meyer und Rowan 1977) \\
\hline
\end{tabular}

\subsubsection{Lose gekoppelte Systeme und organisierte Anarchien}

Möchte man die Gestaltung von Studium und Lehre analysieren, sind zwei Theorien zur Funktionsweise von Hochschulen besonders relevant. So argumentiert Weick (1976, S. 3 ff.) in der Theorie der lose gekoppelten Systeme, dass Hochschulen durch ihre Organisation in Disziplinen und Fächern nur gering miteinander verbunden und voneinander abhängig sind. Einzelne Fachbereiche stellen lose gekoppelte Systeme dar, weil sie überwiegend autonome Entscheidungsstrukturen aufweisen und strukturell gering verflochten sind. Daher kann die Lehre in einem Fachbereich beispielsweise vollkommen unabhängig von der Lehre in einem anderen Fachbereich erfolgen (Hüther und Krücken 2016, S. 175 f.). Diese Zuordnung der Aufgaben zeigt sich auch in der gesetzlichen Zuweisung der Kernaufgaben Forschung und Lehre an die Fakultäten oder Fachbereiche (Land Rheinland-Pfalz $\S 87$ ). Wie die Studienstruktur der Studiengänge gestaltet ist, hängt somit primär von Entscheidungen der Fakultätsoder Fachbereichsräte ab. Da die Mehrheit der Gremienmitglieder in der Regel Professorinnen und Professoren sind, prägt diese Gruppe die Entscheidungen durch ihre Interessen und Meinungen stark (Land Rheinland-Pfalz § 37). Gleichzeitig ist die grundgesetzlich garantierte Freiheit von Forschung und Lehre zu beachten, die den Professorinnen und Professoren eine hohe Selbstbestimmung in den Lehrinhalten und der Didaktik garantiert. Wie stark die Präsidien hier eingreifen können, hängt von der Steuerungsstruktur und der Hochschulkultur ab (siehe Kapitel 9.1.2).

Die Theorie der organisierten Anarchie nach Cohen et al. (1972, S. 1 ff.) weist darauf hin, dass die Entscheidungsträger in Gremien fluktuieren. Die in den 
Gremien verhandelten Themen sind zusätzlich mit konfliktären und uneindeutigen Zielen von Forschung und Lehre hinterlegt (Krücken und Wild 2010, S. 58 f.). Darüber hinaus sind Problemlösungen aufgrund der hohen Komplexität von Forschung und Lehre häufig uneindeutig (Hüther und Krücken 2016, S. 187). So ist der Erfolg einer didaktischen Veränderung - beispielsweise der Einführung von Blended-Learning - von zahlreichen Faktoren, wie der Medienkompetenz von Lehrenden und Studierenden, der Technik, der Lernziele der Lehrveranstaltung sowie der didaktischen Gestaltung der virtuellen Lehre, abhängig. Gleiches gilt für wichtige Ziele im Bereich der Studierbarkeit, wie die Reduktion des Studienabbruchs oder eine hohe Studienzufriedenheit. Es ist aufgrund der vielfältigen Einflussfaktoren auf diese Phänomene nicht möglich, mit einer einzigen klaren Intervention zu einer hohen Studienzufriedenheit oder einer geringen Abbruchquote zu gelangen.

Aus den beiden Theorien lassen sich drei Ansatzpunkte für die Anwendung des Konzeptes struktureller Studierbarkeit ableiten. Erstens sind die einzelnen Fakultäten oder Fachbereiche für die Weiterentwicklung von Studium und Lehre zuständig. Zweitens sind die Professorinnen und Professoren sowohl als Entscheidungsträger in den Gremien als auch als Lehrende anzusprechen. Denn die Weiterentwicklung der Studiengänge und der Lehre obliegt primär ihnen, auch wenn andere Akteure, wie die Studierendenvertretungen und die politischen Entscheidungsträgerinnen und Entscheidungsträger, die Prozesse ebenfalls beeinflussen können. Drittens sollte das Konzept der strukturellen Studierbarkeit für die Entscheidungsträgerinnen und Entscheidungsträgern flexibel sein und als „Brille“ genutzt werden, um der jeweiligen Situation in der Hochschule und dem Fach gerecht zu werden. Reflexionsfragen für eine solche flexible Anwendung des Konzeptes finden sich in Kapitel 10.

\subsubsection{Hochschulgovernance}

Über die Funktionsweisen von Hochschulen hinaus spielt eine wichtige Rolle, wie Hochschulen intern und extern gesteuert werden. Das traditionelle deutsche Steuerungsmodell von Hochschulen ist von einer starken staatlichen Einflussnahme sowie einer starken akademischen Selbstverwaltung geprägt. Die Steuerung innerhalb der Hochschule (1) erfolgt im traditionellen deutschen Modell durch starke Selbstverwaltungsgremien wie Senate und Fakultäts- oder Fachbereichsräte. Was die innere Hierarchie (2) betrifft, sind die Durchgriffsmöglichkeiten der Hochschulleitung und Dekanate im Vergleich zu denen der Führungsebenen von Wirtschaftsunternehmen als eher gering anzusehen. Dafür nehmen die Bundesländer eine staatliche Detailregulierung (3) 
durch Vorgaben vor, beispielsweise bei der hochschulinternen Verteilung von Finanzmitteln oder der Einrichtung von Studiengängen.

Die Governancestrukturen in den Hochschulen haben sich in den letzten Jahren verändert. Hüther und Krücken (2016, 122 ff.) zeichnen eine Entwicklung hin zum New-Public-Management (NPM) nach. Sie verdeutlichen in Tabelle 36 den Unterschied dieser beiden Steuerungsmodelle anhand der fünf Kriterien des Equalizer-Modells von Schimank (Beor et al. 2010, 3 ff.). Das NPM-Konzept sieht anstatt einer direkten Detailsteuerung durch den Staat und die hochschulinternen Gremien eine indirekte Kontextsteuerung vor. Verkürzt ausgedrückt stehen Ziele und Aufgaben im Mittelpunkt, die die Hochschulen sich selbst setzen oder extern vorgegeben werden und von den Hochschulen erreicht werden sollen. Wie dies geschieht, bleibt den Hochschulen überwiegend selbst überlassen. Dabei reduzieren sich staatliche Vorgaben, und es erhöht sich die Außensteuerung durch Zielvorgaben und hochschulexterne Beraterinnen und Berater (4). Gleichzeitig erhöht sich die Steuerung durch Wettbewerb (5) unter den Hochschulen und innerhalb der Hochschulen. Dieser Wettbewerb wird im Bereich der Lehre unter anderem durch die Vergabe von zeitlich begrenzten Zweit- oder Drittmitteln durch den Staat (z. B. Offene Hochschule, Qualitätspakt Lehre, Programmmittel Hochschulpakt) oder durch private Geldgeber (z. B. Stifterverband der deutschen Wirtschaft für die deutsche Wissenschaft) offenkundig. Die Hierarchie in der Hochschule erfährt eine Stärkung, sodass den Hochschulleitungen und Dekanaten mehr und den Gremien weniger Entscheidungskompetenzen zukommen (Schubert 2009, S. 1225). Fachhochschulen fällt laut Nickel (2011, 132 ff.) die Veränderung im Sinne des NPM leichter als Universitäten, da sie durch die Fokussierung auf die Lehre eher nach gemeinschaftlichen Zielen handeln und wirtschaftlichen und gesellschaftlichen Akteuren näherstehen. Bei den all diesen Änderungen ist zu beachten, dass sie von den Hochschulleitungen deutlich positiver bewertet werden als von den Professorinnen und Professoren. Die Professorinnen und Professoren geben häufiger an, dass die neuen Zuständigkeiten zu Konflikten führen würden (Bogumil et al. 2013, 117 ff.). Darüber hinaus verändert eine Strukturänderung nicht mit sofortiger Wirkung auch die Hochschulkultur, sodass viele Hochschulleitungen die ihr verliehene Macht nicht gänzlich ausüben (Kleimann 2015, 42 f.). 
Tabelle $36 \quad$ Vergleich des NPM-Modells mit dem traditionellen deutschen Governancemodell. Eigene Darstellung nach Hüther und Krücken (2016, S. 140)

\begin{tabular}{llll}
\multicolumn{2}{l}{ Kriterien } & $\begin{array}{l}\text { Traditionelles } \\
\text { deutsches Modell }\end{array}$ & $\begin{array}{l}\text { New-Public- } \\
\text { Management-Modell }\end{array}$ \\
\hline 1. & Akad. Selbstverwaltung & Stark & Schwach \\
\hline 2. & Innere Hierarchie & Schwach & Stark \\
\hline 3. & Staatliche Regulierung & Stark & Schwach \\
\hline 4. & Außensteuerung & Schwach & Stark \\
\hline 5. & Wettbewerb & Schwach & Stark
\end{tabular}

Wegen des Föderalismus unterscheiden sich die Bundesländer darin, wie stark NPM Einzug in die Hochschulgesetze gehalten hat. Hüther (2010) analysiert die Hochschulgesetze aus dem Jahr 2008 und zeigt diese Unterschiede anhand der Sach- und Personalkompetenzen von Hochschulen deutlich auf. Der Autor ordnet die Länder Rheinland-Pfalz und Sachsen-Anhalt als Kollegial-Modell mit einem starken Senat bzw. Konzil und einer mittelstarken bis schwachen Hochschulleitung ein. Diese Länder verfolgen also ein eher traditionelles Konzept der Hochschulsteuerung. Hingegen bildet das Hochschulgestz von Hessen das Hierarchiemodell ab, welches von starken Hochschulleitungen und einem relativ schwachen akademischen Senat geprägt wird (Hüther 2010, S. 333). Hessen orientiert sich damit zum Zeitpunkt der Analyse stark am NPM.

Kapitel 9.1.1 weist als Zuständige für die lehr- und studiengangbezogenen Entscheidungen primär die Professorinnen und Professoren aus. Dies gilt insbesondere im traditionellen deutschen System mit einer starken akademischen Selbstverwaltung. In Bundesländern mit einer gestärkten inneren Hierarche im Sinne des NPM hingegen liegt die Verantwortung stärker bei den Dekanats- und Hochschulleitungen, sodass deren Einfluss auf die Studienganggestaltung zunimmt. Diese Zuständigkeiten und Entscheidungsstrukturen sind bei der Weiterentwicklung von Studiengängen zu berücksichtigen.

\subsubsection{Soziologische neoinstitutionalistische Organisationtheorie}

Als weitere Perspektive auf die Berücksichtigung struktureller Studierbarkeit bietet sich die Theorie des soziologischen Neoinstitutionalismus (Meyer und Rowan (1977); DiMaggio und Powell (1983), Übersicht siehe Hasse und Krücken (2005)) an. Diese Theorie ist eine von mehreren neuen Institutionentheorien, wie etwa der Rational-Choice-Institutionalismus (Hall und Taylor 1996) oder der akteurszentrierte Institutionalismus (Scharpf 1997), die Institutionen und deren Rolle in der Gestaltung sozialen Handelns untersuchen. 
Hasse und Krücken (2013, S. 237) verdeutlichen im Sinne des soziologischen Neoinstitutionalismus, dass die Erwartungen einer Gesellschaft die Handlungen von Organisationen und ihren Mitgliedern beeinflussen:

„Individuelle und kollektive Handlungen sind nur im Rahmen allgemein-verbindlicher institutioneller Orientierungen zu verstehen und können auch nur dann entstehen, wenn es diese übergreifenden gesellschaftlichen Erwartungsstrukturen gibt, an denen sich individuelle und kollektive Akteure ausrichten können“ (Hasse und Krücken 2013, S. 237).

Die soziologische neoinstitutionalistische Theorie fokussiert die Handlungen von Organisationen und ihrer Mitglieder. Sie beschäftigt sich unter anderem mit der Frage, durch welche Impulse Organisationen ihre Struktur und ihre Handlungen der Akteure ändern. Dabei orientieren sich Organisationen wie Hochschulen an den Anforderungen ihrer Umwelt, um ihre Arbeit zu legitimieren (Hasse und Krücken 2013, S. 239). In der Umwelt von Hochschulen sind Akteure wie die Politik, Geldgeber sowie die Öffentlichkeit relevant. Die Theorie unterscheidet zwischen einer Formalstruktur und einer Aktivitätsstruktur von Organisationen. Die Formalstruktur ist nach außen sichtbar und zeigt sich in der Organisationsstruktur (Organisationseinheiten, Zuständigkeiten). Die Aktivitätsstruktur hingegen wird durch die Handlungen der Mitglieder der Hochschule gestaltet. Dabei ist es Organisationen möglich, die Regelungen und Vorgaben der Formalstruktur unter Umständen kaum oder nur symbolisch zu befolgen. In diesem Fall ist die Aktivitätsstruktur nur lose mit der Formalstruktur verbunden (Hasse und Krücken 2013, S. 239; Hüther und Krücken 2016, S. 165). Am Beispiel der Einführung von Transferstellen in Hochschulen wird dieses Verhalten von Organisationsmitgliedern deutlich. So wurden beispielsweise aufgrund des Wunsches der Landespolitik nach verstärktem Wissens- und Technologietransfer im vergangenen Jahrzehnt zahlreiche Transferstellen gegründet (Formalstruktur). Trotz der formalen Stellen fand in den untersuchten Fällen der Transfer vielfach ohne Beteiligung der Transferstellen (Aktivitätsstruktur) statt (Krücken 2003, S. 321 ff.). Ein weiteres Beispiel betrifft die Gleichstellung und Forschungsexzellenz. Nach Hüther und Krücken (2016, S. 169) sind

„Geschlechtergleichstellung und Forschungsexzellenz als in der gesellschaftlichen Umwelt von Hochschulen institutionalisierte Mythen [zu] verstehen, die zum Erhalt von Umweltlegitimation von hoher Bedeutung sind und in der Organisation vor allem zum Aufbau von Formalstrukturen führen, während die Ebene der Aktivitätsstruktur, auf der in erheblichem Maße ,business as usual" stattfindet, nur lose hiermit verbunden ist" (Hüther und Krücken 1012, S. 169). 
Da diese Einschätzung das Thema der Heterogenität von Studierenden betrifft, ist eine mögliche Diskrepanz von formaler Struktur und tatsächlicher Umsetzung in den Studiengängen und der Lehre auch beim Thema Studierbarkeit relevant. Die Berücksichtigung von Heterogenität schlägt sich an vielen Hochschulen in der Gründung von Diversity-Abteilungen oder der Beteiligung an Audits zu Diversity und Familienfreundlichkeit wieder (Buß und Buß 2015, S. 98 f.). Eine solche Einrichtung von Abteilungen oder Zertifizierungen bedeuten allerdings nicht automatisch, dass die Belastungen durch Familie, Beruf oder Beeinträchtigungen in den Studienstrukturen Berücksichtigung finden.

Damit Studiengänge für eine vielfältige Studierendenschaft optimal studierbar sind, muss daher die Ebene der Aktivitätsstruktur angesprochen werden. Je nach Governancestruktur sind dabei unterschiedliche Akteure zu berücksichtigen, in der Regel handelt es sich aber insbesondere um die Verantwortlichen für die Studiengänge in den Fakultäten und Fachbereichen. Wenn es um die Einführung von virtueller Lehre und Blockveranstaltungen geht, müssen Lehrende ihre didaktischen Konzepte verändern. Die in den Studiengängen lehrenden Professorinnen und Professoren mit in die Entwicklungen einzubinden $\mathrm{zu}$ holen, erscheint zusammenfassend ein vordringliches Anliegen.

\subsection{Anforderungen an die Umsetzung der strukturellen Studierbarkeit}

Die vorherigen Abschnitte zeigen, dass Professorinnen und Professoren als Gremienmitglieder und Lehrende eine wichtige Rolle in der Entscheidungsfindung und Umsetzung von veränderten Studienstrukturen spielen. Die Leitungsebenen in den Hochschulen können Veränderungen ebenfalls forcieren und sie durch entsprechende Rahmenbedingungen unterstützen. In diesem Abschnitt steht die Frage im Mittelpunkt, welche Anforderungen die Hochschulleitungen sowie ihre Lehrenden und Mitarbeitenden an eine veränderte Studienstruktur stellen und welche Rahmenbedingungen bei Veränderungen zu berücksichtigen sind.

\subsubsection{Anforderungen von Hochschulleitungen an die Umsetzung}

Die Anforderungen von Hochschulen beim Thema Studierbarkeit und Hochschulöffnung lassen sich aus politischen und hochschulischen Zielsetzungen auf der einen und den zur Verfügung stehenden Ressourcen auf der anderen Seite ableiten. Diese beiden Aspekte werden im Folgenden analysiert, um die 
Anforderungen der Leitungsebenen von Hochschulen an die Anwendung des Konzeptes struktureller Studierbarkeit zu umreißen.

Zunächst stehen daher die strategischen Ziele von Hochschulen in Bezug auf Studierbarkeit und Hochschulöffnung im Mittelpunkt. Die Hochschulziele werden im Sinne der neoinstitutionalistischen Theorie durch die Politik beeinflusst. Der weite Begriff der Politik bezieht sich dabei insbesondere auf die gesetzgebende Landesebene, aber auch Akteure ohne direkte Gesetzgebungskompetenz wie die Kultusministerkonferenz (KMK), das Bundesministerium für Bildung und Forschung (BMBF), den Wissenschaftsrat oder den Akkreditierungsrat. Die Verbesserung der Studierbarkeit für eine heterogene Studierendenschaft wurde in den letzten Jahren insbesondere unter dem Schlagwort der „Hochschulöffnung“ diskutiert. Bisher liegt allerdings wenig Literatur zu der Frage vor, welche Ziele die Politik zur Hochschulöffnung und der Studierbarkeit konkret nennt. Lediglich Mergner und Bosse (2018, S. 62) analysieren die Ziele politischer Akteure im Bereich der Hochschulöffnung anhand von Dokumentenanalysen. Sie ziehen hierfür Dokumente des BMBF, der KMK und des Wissenschaftsrats heran. Die Autorinnen zeigen, dass eine breite Teilhabe an Hochschulbildung und die Erhöhung bzw. Beibehaltung der Studierendenzahlen die Hauptziele der politischen Akteure darstellen. Die Teilhabe betrifft dabei nicht nur den Hochschulzugang, sondern auch den Studienerfolg. Dieser steht auch bei der strukturellen Studierbarkeit im Mittelpunkt.

Doch inwieweit werden diese Ziele von den Hochschulen aufgenommen und umgesetzt? Die neoinstitutionalistische Organisationstheorie nimmt an, dass die Hochschulen sich zum Zweck der Legitimation an den politisch formulierten Zielen orientieren. Da gleichzeitig eine hohe Autonomie der einzelnen Hochschulen und Fachbereiche besteht, können diese jedoch die Zielvorgaben anpassen. Somit ist $\mathrm{zu}$ erwarten, dass die Hochschulen die strukturelle Studierbarkeit unterschiedlich stark in den Blick nehmen. Hochschulen zeigen öffentlichkeitswirksam wichtige strategische Ziele in Leitbildern auf. Gleichzeitig setzen sie in den Leitbildern Akzente, die das Lehr- und Studienverständnis der Hochschule in Ansätzen sichtbar machen. Bisher liegen keine Analysen zu den Hochschulzielen im Bereich Studierbarkeit und Hochschulöffnung auf der Grundlage von Leitbildern vor. Einen ersten Einblick in diese Ziele bietet eine durch die Autorin durchgeführte Leitbildanalyse der Hochschulen in RheinlandPfalz. Im Januar 2018 liegen hochschulweite Leitbilder von acht der elf öffentlichen Hochschulen des Landes vor: der Universität und der Hochschule Mainz, der Hochschule Trier, der Hochschule Koblenz, der Hochschule Kaiserslautern, der Technischen Hochschule Bingen, der Hochschule Worms und der Hochschule Ludwigshafen am Rhein. Da die strukturelle Studierbarkeit sich an den Bedarfen einer heterogenen Studierendenschaft orientiert, werden zunächst 
die Berücksichtigung von Diversität bzw. die Förderung von Teilhabe durch die Hochschulen sowie die Vereinbarkeit von Studium, Familie und Beruf als Kriterien in die Analyse aufgenommen. Studienstrukturen sind aufgrund ihres starken Konkretisierungsgrads in der Regel nicht Teil von strategischen Leitbildern. Die Strukturen sollen aber die Autonomie von Studierenden ermöglichen, damit diese flexibel studieren können. Außerdem wird analysiert, ob Hochschulen explizit die Anpassung der Studiengänge an die Bedarfe der Studierenden benennen. Schließlich geht die Förderung des Studienerfolgs in die Analysen ein.

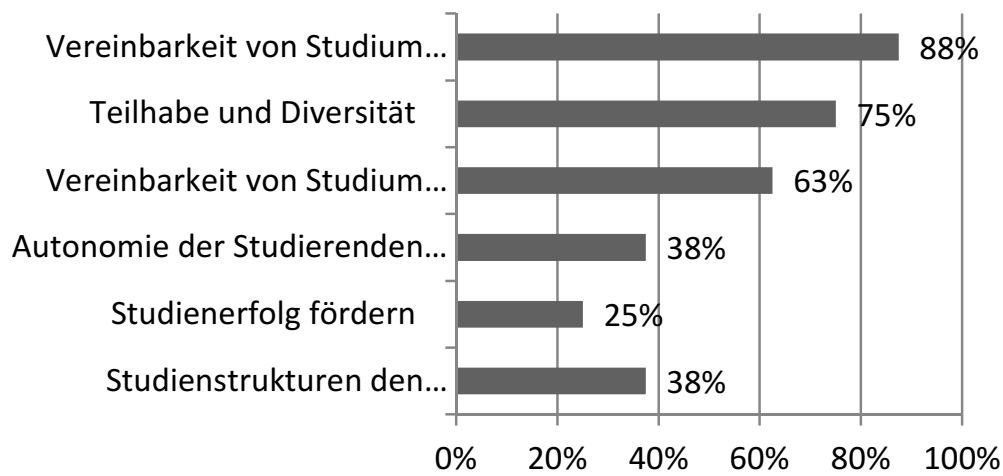

Abbildung 20 Leitbildanalyse von acht rheinland-pfälzischen Hochschulen. Eigene Darstellung.

Abbildung 20 weist aus, welcher Anteil der Hochschulen in ihrem Leitbild das entsprechende Ziel nennt. Dabei werden die Vereinbarkeit von Studium, Beruf und Familie sowie die Berücksichtigung der Diversität und die Teilhabe an Hochschulbildung besonders häufig genannt. Die Hochschulen berücksichtigen somit auch in ihren Leitbildern die von Mergner und Bosse (2018) herausgearbeiteten hochschulpolitischen Ziele der Teilhabe und des Studienerfolgs dieser diversen Studierendenschaft und legitimieren somit ihr Handeln. Etwa ein Drittel der Hochschulen möchte ihre Ziele im Bereich der Vereinbarkeit und Diversität durch die Anpassung von Studienstrukturen erreichen und gleichzeitig die Autonomie der Studierenden fördern. Die Ergebnisse zeigen, dass die rheinland-pfälzischen Hochschulen durchaus die Ziele unterstützen, die mit einer strukturellen Studierbarkeit verbunden sind. Die konkrete Umsetzung in den Studienstrukturen wird selten thematisiert. Dies kann jedoch auch an dem Dokumententyp des Leitbildes liegen, das als Teil der Formalstruktur strategische Ziele abbildet. Inwiefern die Hochschulen ihre Leitbilder umsetzen (Aktivitätsstruktur), bleibt unklar. 
Hinweise für die Umsetzung von Leitbildern bieten die Projektbeschreibungen von Hochschulen, die im Qualitätspakt Lehre aktiv sind. Die Hochschulen dokumentieren in den Projektbeschreibungen ihr Ziel, den Studienerfolg der heterogenen Studierendenschaft $\mathrm{zu}$ verbessern. Viele Dokumente weisen jedoch auch auf die Herausforderungen hin, vor denen die Hochschulen aufgrund der hohen Studierendenzahlen und der diversen Studierendenschaft stehen (Mergner und Bosse 2018, S. 59). Hanft (2015, S. 23) analysiert ebenfalls anhand des Qualitätspakts Lehre, welche Maßnahmen Hochschulen durchführen. Diese zielen insbesondere auf die Verbesserung der Infrastruktur, der Personalausstattung und der Qualifizierung des Personals ab. Die Themen Hochschulöffnung bzw. Durchlässigkeit sowie Diversität werden seltener genannt.

Finanzielle, infrastrukturelle und personelle Rahmenbedingungen können ein Hindernis für die Umsetzung einer Studienstruktur darstellen, die auf eine hohe Flexibilität setzt. Denn die Entwicklung von virtueller Lehre oder die Einführung von Wahlmöglichkeiten benötigt freie personelle Ressourcen und freie Lehrdeputate. Führen Hochschulen neue Studienformate wie das Teilzeitstudium ein, benötigen sie personelle Ressourcen zur Beratung und Betreuung der Studierenden (Steinhardt 2011a, S. 8). Flexible Strukturen bieten jedoch auch Vorteile für Hochschulen, wenn jene beispielsweise durch virtuelle Lehre die räumlichen Ressourcen weniger nutzen oder Blockveranstaltungen auf Tage mit geringer Raumauslastung legen.

\subsubsection{Anforderungen von Lehrenden und Mitarbeitenden an die Umsetzung}

Die Anforderungen von Professorinnen und Professoren sowie Mitarbeitenden werden von persönlichen Einstellungen, vom Selbstverständnis des Faches sowie von der Bedeutung der Veränderung für die betroffenen Personen und für die Lehrsituation beeinflusst (Alheit 2009, S. 217; Hall und Hord 2014, S. 136 f.). Die Fachkultur nimmt bei der Gestaltung der Studienstrukturen und der Lehre eine wichtige Rolle ein. Sie beeinflusst, welche Lehrformate (z. B. Seminar, Vorlesung), Prüfungen und zeitliche Formate (wöchentliche Veranstaltungen oder im Block) üblich sind und wie offen Lehrende gegenüber digitalen Medien sind (Huber 2011, S. 110 ff.; Kämper 2014, S. 118, 126). In ihrem Werk „Academic Tribes and Territories" analysieren Becher und Trowler (2011, S. 34 ff.), ob Fächer sich überwiegend auf messbare, reproduzierbare Daten beziehen - dann handelt es sich um „harte“ (im Gegensatz zu „weichen“) Wissenschaften - oder ob sie einen starken Anwendungsbezug haben, dann handelt es sich um „reine“ Wissenschaften mit geringem oder ,angewandte“ Fächer mit hohem Anwendungsbezug. Alheit (2009, S. 217 ff.) bezieht diese Einordnung auf die 
Einstellungen zur Hochschulöffnung beziehungsweise gegenüber nichttraditionellen Studierenden. Der Autor zeigt, dass Lehrende von anwendungsorientierten Fächern - hierunter fallen beispielsweise die Soziale Arbeit oder die Ingenieurswissenschaften - ein tendenziell inklusiveres Verständnis von der Hochschule haben. Jütte et al. (2017, S. 69) führen ergänzend das Beispiel der juristischen Fakultät auf, die als eher selektierend beschrieben wird. Hat ein Fach ein eher inklusives Selbstverständnis, dann steht es der Weiterentwicklung seiner Strukturen im Sinne struktureller Studierbarkeit möglicherweise offener gegenüber. Selbstverständlich sind aber auch individuelle Einstellungen wirksam: so können Lehrende oder Gruppen von Lehrenden eine von der Fachkultur abweichende Meinung vertreten.

Neben den Einstellungen gegenüber der Hochschulöffnung spielt auch eine Rolle, wie stark die Person von der jeweiligen Änderung betroffen ist. Mit der Hochschulöffnung und der Veränderung von Studienstrukturen sind viele Befürchtungen verbunden. Lehrende und Mitarbeitende befürchten, dass die Bedarfe einer diversen Studierendenschaft Mehrarbeit für sie mit sich bringen. In diesem Kontext geben Mitarbeitende an, dass ihnen personelle Ressourcen für zusätzliche Betreuung und Beratung fehlen würden. Die zeitliche Flexibilität von Lehrenden ist gefragt, wenn Studierende Lehrveranstaltungszeiten außerhalb der bisher üblichen Zeiten benötigen (Müller et al. 2017, S. 217). Bezogen auf die Lehre befürchten einige Professorinnen und Professoren außerdem, dass das akademische Niveau sinken und die hochschulische Sozialisation leiden könnte. Als Grund hierfür werden die geringeren zeitlichen Ressourcen von Studierenden mit familiären oder beruflichen Belastungen genannt. Lehrende erwarten von der Hochschulöffnung jedoch auch positive Effekte. Sie sehen die nicht-traditionellen und beruflich qualifizierten Studierenden als hoch motivierte Studierendengruppe an, von deren beruflichen Erfahrungen sie selbst und die anderen Studierenden profitieren können (Müller et al. 2017, 218).

Neben dieser Forschung zu Einstellungen existiert nur wenig Literatur zu den Auswirkungen, welche die Implementierung von neuen Studienstrukturen auf die Hochschullehrenden und Mitarbeitenden haben. Lediglich zum Blended-Learning liegt ein umfangreiches Literaturkorpus vor. Darin wird vorrangig der zeitliche Aspekt bei der Erstellung und Durchführung virtueller Lehre diskutiert, wobei dieser sowohl vor- als auch nachteilig gesehen wird. So ist die Erstellung des Lehrkonzeptes für virtuelle Lehre einerseits aufwendig. Neben dem Aufwand sind viele Lehrende einer Unsicherheit ausgesetzt, da hochschulintern unklar ist, wie die Erstellung und Durchführung virtueller Lehre auf das Deputat angerechnet wird (Lungershausen et al. 2016, S. 103; Schmahl 2008, S. 93). Andererseits ist ein Hauptgrund für die Einführung virtueller Lehre, dass Präsenzveranstaltungen durch virtuelle Lehre ersetzt werden und Lehrende und Studierende dadurch 
zeitlich und örtlich flexibel sein können. Da aus Sicht vieler Akteure die organisatorischen und didaktischen Vorteile der virtuellen Lehre überwiegen, wird die Einführung von Blended-Learning von vielen Hochschulen forciert. Dabei sollten den Lehrenden gute technisch-didaktische Unterstützung sowie eine einfach zu bedienende Lernplattform zur Verfügung stehen (Kreidl 2011, 86 ff.). Neben der zeitlichen Komponente sind die Lehrenden bei der Nutzung von Blended-Learning persönlich betroffen, da sie hierfür mediendidaktische Kompetenzen benötigen. Verfügen Lehrende über diese Kompetenz, ist es ihnen möglich, „Medien auf ihre didaktische Eignung hin einzuschätzen und zugänglich zu machen" (Schmahl 2008, S. 41). Auf der medientechnischen Ebene können Lehrende Medien selbst anwenden und erweitern, also zum Beispiel Lernplattformen bedienen oder Screencasts erstellen. Darüber hinaus sollen Lehrende die Studierenden bei der Nutzung der virtuellen Lehre und der Selbststeuerung der Lernprozesse anleiten und sie webgestützt beraten können. Schließlich sollen sie ihre Aktivitäten urheberrechtlich und datenschutzrechtlich einschätzen können (Horvath 2009, S. 3). Über die Kompetenz, mit diesen komplexen Anforderungen umzugehen, verfügen nicht alle Lehrenden. Dies kann zu Unsicherheit und Unklarheit führen, wenn es darum geht, was die Einführung virtueller Lehre bedeutet und wie sie durch die Lehrpersonen geleistet werden kann. E-didaktische Qualifizierungen und die individuelle Unterstützung bei der didaktischen Konzeption sowie bei der technischen Umsetzung sind daher grundlegend für die erfolgreiche Einführung von Blended-Learning in der Hochschule (Thillosen und Hansen 2009, S. 135 f.).

Neben der Einführung von Blended-Learning stehen in dieser Arbeit die Strukturelemente der Lehrveranstaltungszeiten, der Wahlmöglichkeiten sowie der Studienformate im Mittelpunkt. Aus Diskussionen im Projekt „Offenes Studienmodell Ludwigshafen" ist deutlich geworden, dass bei der Einführung von Blockveranstaltungen die zeitlichen Präferenzen der Lehrenden zu berücksichtigen sind. Gleichzeitig sollten Lehrende selbst entscheiden, welche Veranstaltungen sich didaktisch für eine Durchführung im Blockformat eigenen. Dieser inhaltlich-didaktische Aspekt spielt auch bei der Einführung von Wahlmodulen eine wichtige Rolle, da nur die Lehrenden über sinnvolle thematische Ausweitungen entscheiden können. Somit sind beide Studienstrukturen sowohl von der Verwaltungsseite in Bezug auf Ressourcen und Organisation zu prüfen als auch inhaltlich durch die Lehrenden zu bewerten. Ähnliches gilt für die Einführung neuer Studienformate wie das Teilzeitstudium, da die Lehrenden bei der inhaltlichen Gestaltung sowie der Beratung und Betreuung mitwirken. 
Open Access Dieses Kapitel wird unter der Creative Commons Namensnennung 4.0 International Lizenz (http://creativecommons.org/licenses/by/4.0/deed.de) veröffentlicht, welche die Nutzung, Vervielfältigung, Bearbeitung, Verbreitung und Wiedergabe in jeglichem Medium und Format erlaubt, sofern Sie den/die ursprünglichen Autor(en) und die Quelle ordnungsgemäß nennen, einen Link zur Creative Commons Lizenz beifügen und angeben, ob Änderungen vorgenommen wurden.

Die in diesem Kapitel enthaltenen Bilder und sonstiges Drittmaterial unterliegen ebenfalls der genannten Creative Commons Lizenz, sofern sich aus der Abbildungslegende nichts anderes ergibt. Sofern das betreffende Material nicht unter der genannten Creative Commons Lizenz steht und die betreffende Handlung nicht nach gesetzlichen Vorschriften erlaubt ist, ist für die oben aufgeführten Weiterverwendungen des Materials die Einwilligung des jeweiligen Rechteinhabers einzuholen.

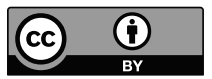

